\title{
Road to comprehensive estimation of antimicrobial resistance (AMR) disease burden in Japan
}

\author{
Shinya Tsuzuki ${ }^{1,2, *}$, Nobuaki Matsunaga ${ }^{1}$, Norio Ohmagari ${ }^{1,3}$ \\ ${ }^{1}$ AMR Clinical Reference Center, National Center for Global Health and Medicine, Tokyo, Japan; \\ ${ }^{2}$ Faculty of Medicine and Health Sciences, University of Antwerp, Antwerp, Belgium; \\ ${ }^{3}$ Disease Control and Prevention Center, National Center for Global Health and Medicine, Tokyo, Japan.
}

\begin{abstract}
Antimicrobial resistance (AMR) is currently a global health threat. Many countries have issued their own national action plans following the publication of the Global Action Plan on AMR by the World Health Organization. The government of Japan established its own National Action Plan on AMR in 2016; however, Japan's AMR countermeasures are still in the developmental stage. Recently, the AMR Clinical Reference Center (AMR CRC) in Japan estimated the disease burden of AMR in the form of number of deaths attributed to blood-stream infections caused by antimicrobial-resistant organisms. However, a more extensive and precise assessment is needed to understand the disease burden of AMR more clearly and enable us to compare these indicators with those published by other countries. Cassini and colleagues from the World Health Organization estimated the disease burden of AMR in the European Union as disability-adjusted life years (DALYs) in 2018. Their study could be considered an important milestone in terms of its thoroughness. If we hope to estimate the disease burden of AMR in a more precise manner, age-stratified patient data is needed in conjunction with a surveillance report. At present, AMR CRC is attempting to establish such data for examination at the national level - a challenging but worthwhile task.
\end{abstract}

Keywords: antimicrobial resistance, DALYs, surveillance

\section{Introduction}

Antimicrobial resistance (AMR) is currently one of the greatest global health issues $(1,2)$. The Japanese government published the National Action Plan on AMR in 2016 (3), following the publication of the Global Action Plan by the World Health Organization (WHO) (1). Subsequently, the AMR Clinical Reference Center (AMR CRC) was established as the main hub of AMR countermeasures in Japan in 2017 (4). As a part of the National Action Plan, AMR CRC has conducted epidemiological research related to this area.

Appropriate evaluation of the disease burden of AMR is needed to prepare appropriate countermeasures. The Japan Nosocomial Infections Surveillance (JANIS) program, which is organized by the Ministry of Health, Labour and Welfare, provides basic information on the incidence and prevalence of nosocomial infections and antimicrobial-resistant bacteria (5). The JANIS Clinical Laboratory module collects all routine microbiological test results from approximately 2,000 hospitals that voluntarily participate in the surveillance and account for a quarter of the total of approximately 8,000 hospitals across Japan. However, the disease burden of AMR is composed not only of the incidence of infections and proportion of resistance but also of other clinical and socioeconomic aspects. In this sense, surveillance data from JANIS is not tied to the individual data that have been collected to date and are thus insufficient for estimating the precise disease burden of AMR. The National Epidemiological Surveillance of Infectious Diseases Program is also a national surveillance system based on reporting from physicians and veterinarians and includes information about AMR (6). However, its main objective is to grasp the trends of the epidemiology of infectious diseases in accordance with the Act on the Prevention of Infectious Diseases and Medical Care; it is not designed to analyze the AMR disease burden.

Tsuzuki and colleagues estimated the disease burden of blood-stream infections (BSIs) caused by methicillinresistant S. aureus (MRSA) and fluoroquinolone-resistant E. coli (FQREC) via number of deaths (7). However, as they stated, number of deaths is not the best proxy for the disease burden of AMR. Moreover, their results included methodological limitations due to the difficulty in correlating a surveillance report with individual clinical/socioeconomic data. Further research is required to estimate the total disease burden of AMR in Japan. In this article, we will discuss how to overcome these limitations and establish a robust and comprehensive 
evaluation framework for AMR disease burden, which will enable us to understand its more precise societal impact and provide a basis for comparison with other countries.

\section{Achievements in a previous study}

There is room for discussion about what constitutes an appropriate indicator for the assessment of the comprehensive disease burden of AMR. Considering that AMR is a global health threat not limited to developed countries, disability-adjusted life years (DALYs) is one promising candidate as a single indicator of disease burden. In particular, the Global Disease Burden project has made great progress in this area and therefore supports health-care policy making $(8,9)$. Cassini and colleagues from the WHO estimated the disease burden of AMR in European Union (EU) countries in the form of DALYs in 2018 (10). If we can estimate the disease burden of AMR in a similar way, it would constitute an important step toward AMR countermeasures in Japan because international comparisons will provide beneficial insights.

The excellence of Cassini et al.'s work is shown by their comprehensiveness in providing a complex of multiple research projects. First, it should be noted that the use of DALYs has been regarded as problematic in terms of its application to infectious diseases (11). Cassini et al. used DALYs values estimated by the Burden of Communicable Diseases in Europe (BCoDE) project (12), which was launched by the European Center for Disease Prevention and Control in 2009. The BCoDE project used a pathogen-based incidence approach to generate estimates to fully consider all chronic and longterm sequelae related to causative organisms. Their new methodology enabled them to deal with the risk of underestimation to some extent.

Cassini et al. used extensive data from EU countries and the European economic area (EEA) obtained from the European Antimicrobial Resistance Surveillance Network (13), the latter of which has provided agestratified, country-specific data, thereby enabling users to not only compare situations among EU/EEA countries but also estimate the years of life lost (YLL) in a more appropriate manner.

Cassini et al. also evaluated years lost due to disability (YLD) in a detailed manner. Their evaluation of YLD is based on another article published in 2018 based on the results of the BCoDE project (14). They included not only disability attributed to infectious diseases but also length of stay (LOS) in hospitals due to infectious diseases in the context of YLD. For example, they calculated disability from BSIs and multiplied that by mean LOS of BSIs. By using this method, the burden of AMR can be seen more clearly because LOS attributed to infectious diseases caused by antimicrobial resistant organisms tends to be longer. Additionally,
Colzani and colleagues developed a new freely available toolkit for DALYs calculation in the BCoDE project (15). This toolkit made their disease models openly accessible to everyone, even from outside EU/EEA countries.

\section{Limitation of the current evidence from Japan}

As mentioned above, AMR CRC published the first evidence on the disease burden of AMR in Japan last December (7). That work can be appreciated because it provides indicators for Japanese healthcare policy makers. Nevertheless, its limitations should be considered and further evaluation is required to fully understand the disease burden of AMR in Japan.

The limitations of our work can be observed more clearly when we consider differences between our work and that of Cassini et al.. Our work focused on the number of deaths attributable to BSIs caused by MRSA and FQREC. These two strains account for the largest part of the disease burden of AMR and BSIs are the main cause of death due to infectious diseases. Nevertheless, this work does not explain the "total" disease burden of AMR because it does not include diseases other than BSIs, clinical outcomes other than death, and organisms other than MRSA and FQREC.

In addition, we should take note that several methodological challenges have not yet been resolved. We estimated the number of deaths due to BSIs in a similar way to Cassini et al.. We used the case fatality ratio derived mainly from two domestic studies $(16,17)$. However, these studies are largely based on a cohort of tertiary care hospitals; therefore, it is difficult to reflect heterogeneity among different level facilities. In Japan, it is generally difficult to find evidence on the epidemiology of infectious diseases such as mortality from BSIs. Furthermore, we could not find any data on the prevalence of sequelae caused by infectious diseases, LOS due to AMR, and so forth.

\section{Future challenges}

As explained above, evaluation of disease burden brought about by AMR needs to be more precise and comprehensive. However, there is a large gap between our ideals and reality. We will have to bridge this gap within the next few years. Many types of data and evidence will be necessary to construct our own framework for evaluating AMR disease burden. The list of required data and evidence is as follows:

i) Age-, disease-, and organism-specific incidence, mortality, and morbidity (e.g., incidence of MRSA pneumonia in infants, mortality of FQREC BSIs in elderly, etc.);

ii) Age-, disease-, and organism-specific LOS (LOS of MRSA BSIs, etc.);

iii) Disability weight of infectious diseases 


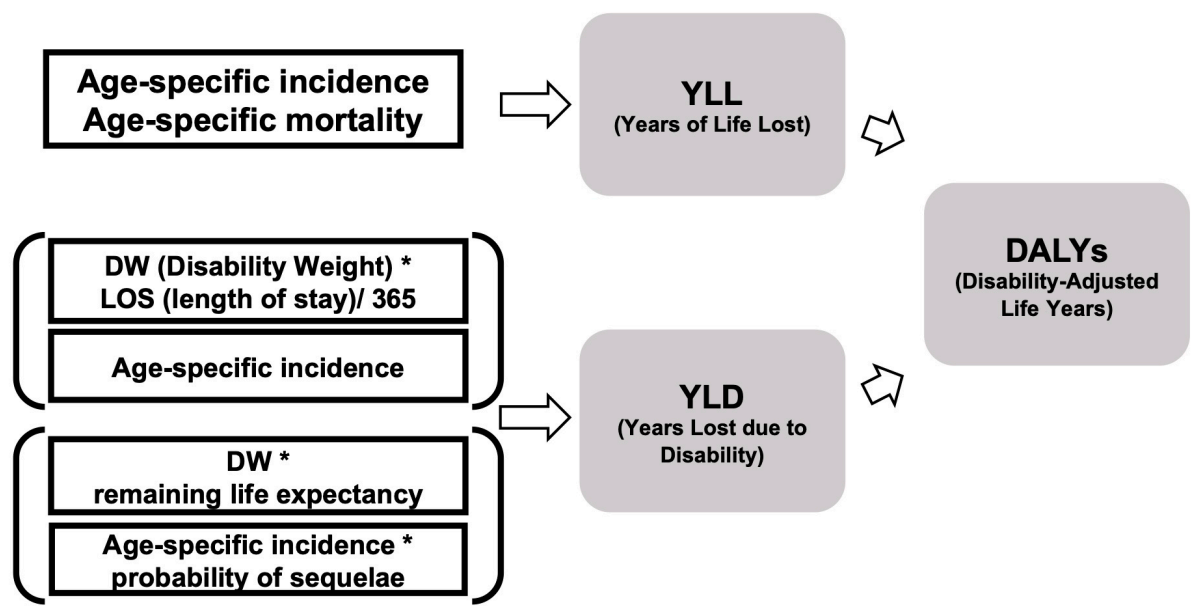

Figure 1. A flowchart regarding the processes in which required data and evidence turn into indicators of disease burden.

(pneumonia, BSIs, etc.);

iv) Disability weight of sequelae due to infectious diseases (cognitive impairment, physical impairment, etc.).

A flowchart regarding the processes in which required data and evidence turn into indicators of disease burden is presented in Figure 1.

DALYs are represented as the sum of YLL and YLD, so age-specific incidence and mortality data are used to calculate YLL. One of the strengths of the previous study by Cassini et al. is their elaborate review process of mortality estimation (10). Ideally, we would also combine results of multiple studies to reflect on the uncertainty and heterogeneity of probabilities such as mortality. The same can be said of morbidity when estimating the burden of sequelae.

It is noteworthy that LOS in Japanese healthcare facilities seems to be longer than that in other developed countries. For example, Muramatsu and Liang reported that the average LOS in Japan was 2.8 times longer than that in the United States (18). Although the recommended duration of treatment is usually defined among infectious diseases, it is merely a guide and it is possible that LOS in Japan is indeed longer than in other countries; therefore, domestic data is desirable if we hope to estimate more appropriate YLD.

Establishing our own value for disability weight is desirable because quality of life will be different among people in different situations even if they have the same disease or disability (19-21).

At present, we are preparing for what must be done. JANIS recently published age-specific AMR incidence levels. AMC CRC established the AMR One Health Platform, which offers age and specimen stratified AMR data at the national level (22). The national receipt database Open Data Japan (23) provides individual health insurance claim data, which might enable exploration of the prevalence of sequelae caused by infectious diseases.

Much work remains in terms of epidemiological evidence. One of our aims is to encourage Japanese clinicians and epidemiologists to conduct novel research projects that reveal the actual mortality and LOS due to AMR, and hopefully involve different levels of facilities (primary, secondary, and tertiary).

Finally, we should not forget that Cassini et al. are also halfway toward realizing a more precise, comprehensive evaluation of AMR disease burden. For instance, among their stated limitations, they neither considered age-specific mortality when they calculated YLL nor statistical methods in their review process of previous studies (10). If we could include YLL calculated by age-specific mortality and estimate mortality with robust statistical methods, it would be invaluable for our future research. In addition, the research group has not yet attempted to estimate the economic impact of AMR. Clearly, longer LOS and higher treatment costs due to AMR may be considered as an economic burden on our society. If we could conduct cost-effectiveness or costbenefit analysis of AMR at the national level, the results would be of notable value for health policy decision makers.

As described above, we currently face many challenges on our way to realizing a more precise and comprehensive evaluation of AMR disease burden. Although they are difficult to deal with, it is worth tackling them simultaneously because reliable indicators of AMR are needed for health policy making in Japan. We have already been putting forth more effort and believe that we will overcome these challenges in the near future.

\section{Acknowledgements}

We would like to thank Yusuke Asai for his useful suggestions. This work was supported by a Ministry of Health, Labour and Welfare (MHLW) research grant of Japan (H29-shinkougyousei-shitei-005). The funders had no role in the preparation of the manuscript. 


\section{References}

1. World Health Organization. Global Action Plan on Antimicrobial Resistance. https://apps.who.int/ iris/bitstream/handle/10665/254352/sea-cd-308. $p d f ?$ sequence $=1 \& i$ s Allowed $=y$ (accessed January 6, 2020).

2. European Commission. A European One Health Action Plan against Antimicrobial Resitance (AMR). https:// ec.europa.eu/health/amr/sites/health/files/antimicrobial resistance/docs/amr_2017_action-plan.pdf (accessed January 6, 2020).

3. The Government of Japan. National Action Plan on Antimicrobial Resistance (AMR) 2016-2020. https:// www.mhlw.go.jp/file/06-Seisakujouhou-10900000Kenkoukyoku/0000138942.pdf (accessed January 6, 2020).

4. National Center for Global Health and Medicine. AMR Clinical Reference Center. http://amrcrc.ncgm. go.jp/030/010/index.html (accessed January 6, 2020). (in Japanese)

5. Ministry of Health Labour and Welfare Japan. Japan Nosocomial Infections Surveillance (JANIS). https://janis. mhlw.go.jp/english/index.asp (accessed January 6, 2020).

6. Watanabe A, Shimada T, Takahashi T, Arima Y, Kinoshita H, Saitoh T, Kanou K, Matsui T, Sunagawa T, Tanaka-Taya K, Oishi K. Correlates of laboratoryconfirmed measles in Japan, 2011-2015. Vaccine. 2019; 37:1756-1762.

7. Tsuzuki S, Matsunaga N, Yahara K, Gu Y, Hayakawa K, Hirabayashi A, Kajihara T, Sugai M, Shibayama K, Ohmagari N. National trend of blood-stream infection attributable deaths caused by Staphylococcus aureus and Escherichia coli in Japan. J Infect Chemother. 2020; 26:367-371.

8. Murray CJ, Lopez AD. Global mortality, disability, and the contribution of risk factors: Global Burden of Disease Study. Lancet. 1997; 349:1436-1442.

9. Murray CJ, Vos T, Lozano R, et al. Disability-adjusted life years (DALYs) for 291 diseases and injuries in 21 regions, 1990-2010: a systematic analysis for the Global Burden of Disease Study 2010. Lancet. 2012; 380:21972223.

10. Cassini A, Högberg LD, Plachouras $\mathrm{D}$, et al. Attributable deaths and disability-adjusted life-years caused by infections with antibiotic-resistant bacteria in the EU and the European Economic Area in 2015: a population-level modelling analysis. Lancet Infect Dis. 2019; 19:56-66.

11. Zou S. Applying DALYs to the burden of infectious diseases. Bull World Health Organ. 2001; 79:267-269.

12. Kretzschmar M, Mangen MJ, Pinheiro P, Jahn B, Fevre EM, Longhi S, Lai T, Havelaar AH, Stein C, Cassini A, Kramarz P; BCoDE consortium. New methodology for estimating the burden of infectious diseases in Europe. PLoS Med. 2012; 9:e1001205.

13. European Centre for Disease Prevention and Control. About the network. https://www.ecdc.europa.eu/en/aboutus/networks/disease-networks-and-laboratory-networks/ ears-net-about (accessed January 6, 2020).

14. Cassini A, Colzani E, Pini A, Mangen MJ, Plass D, McDonald SA, Maringhini G, van Lier A, Haagsma JA, Havelaar AH, Kramarz P, Kretzschmar ME, On Behalf
Of The BCoDE Consortium. Impact of infectious diseases on population health using incidence-based disabilityadjusted life years (DALYs): results from the Burden of Communicable Diseases in Europe study, European Union and European Economic Area countries, 2009 to 2013. Euro Surveill. 2018; 23:17-00454.

15. Colzani E, Cassini A, Lewandowski D, Mangen M-JJ, Plass D, McDonald SA, van Lier A, Haagsma JA, Maringhini G, Pini A, Kramarz P, Kretzschmar ME. A software tool for estimation of burden of infectious diseases in Europe using incidence-based disability adjusted life years. PLoS One. 2017; 12:e0170662.

16. Takeshita N, Kawamura I, Kurai H, Araoka H, Yoneyama A, Fujita T, Ainoda Y, Hase R, Hosokawa N, Shimanuki $\mathrm{H}$, Sekiya N, Ohmagari N. Unique characteristics of community-onset healthcare- associated bloodstream infections: a multi-centre prospective surveillance study of bloodstream infections in Japan. J Hosp Infect. 2017; 96:29-34.

17. Nagao M. A multicentre analysis of epidemiology of the nosocomial bloodstream infections in Japanese university hospitals. Clin Microbiol Infect. 2013; 19:852-858.

18. Muramatsu N, Liang J. Hospital length of stay in the United States and Japan: a case study of myocardial infarction patients. Int J Health Serv. 1999; 29:189-209.

19. Brazier JE, Fukuhara S, Roberts J, Kharroubi S, Yamamoto Y, Ikeda S, Doherty J, Kurokawa K. Estimating a preferencebased index from the Japanese SF-36. J Clin Epidemiol. 2009; 62:1323-1331.

20. Husereau D, Drummond M, Petrou S, Carswell C, Moher D, Greenberg D, Augustovski F, Briggs AH, Mauskopf J, Loder E; ISPOR Health Economic Evaluation Publication Guidelines-CHEERS Good Reporting Practices Task Force. Consolidated Health Economic Evaluation Reporting Standards (CHEERS) - explanation and elaboration: a report of the ISPOR Health Economic Evaluation Publication Guidelines Good Reporting Practices Task Force. Value Health. 2013; 16:231-250.

21. Brazier J, Ara R, Azzabi I, Busschbach J, ChevrouSéverac H, Crawford B, Cruz L, Karnon J, Lloyd A, Paisley S, Pickard AS. Identification, review, and use of health state utilities in cost-effectiveness models: an ISPOR good practices for outcomes research task force report. Value Health. 2019; 22:267-275.

22. AMR One health platform. https://amr-onehealthplatform.ncgm.go.jp/(accessed January 6, 2020).

23. Ishikawa KB. Medical big data for research use: current status and related issues. Japan Med Assoc J. 2016; 59:110-124.

Received January 14, 2020; Revised April 23, 2020; Accepted May 4, 2020

Released online in J-STAGE as advance publication May 11, 2020.

*Address correspondence to:

Shinya Tsuzuki, AMR Clinical Reference Center, National Center for Global Health and Medicine, 1-21-1 Toyama, Shinjuku-ku, Tokyo 162-8655, Japan.

E-mail: stsuzuki@hosp.ncgm.go.jp 